\title{
On a Class of Gaussian Distributions in Functional Space
}

\author{
A.D. Egorov and A.V. Zherelo \\ National Academy of Sciences, Institute of Mathematics \\ Minsk, Belarus
}

\begin{abstract}
An analog of reproducing Hilbert space of measure for a class of signed Gaussian distributions in the space of functionals is considered. Orthonormal bases in two special cases are constructed.
\end{abstract}

Keywords: Gaussian distributions, Reproducing Hilbert space of measure, Orthonormal bases, Nongaussian sign measure.

\section{Introduction}

In many problems concerning statistics of stochastic processes absolute continuity or singularity of distributions play important role. In case of gaussian processes the reproducing Hilbert spaces of distributions are used in order to formulate necessary conditions of singularity and to write down mutual densities of measures under considerations Rozanov (1968). Moreover, explicit view of orthonormal basis in these spaces are used. In Egorov (1997) an approach was developed to investigation of structure of the space $L_{2}(X, \mu)$ over linear topological space $X$ with nongaussian measure $\mu$ defined by characteristic functional. The approach is based on $L_{2}$-isomorphism $\widehat{S}$ from $L(P) \subset L_{2}(X, \mu)$ into $L_{2}(Y, \widehat{\mu})$, where $Y=L_{2}(X, \gamma), \gamma$ is an arbitrary fixed gaussian measure and $\widehat{\mu}$ is a special gaussian measure on $Y$ defined by $\mu$. So that one can hope for applying some analog of gaussian constructions to some problems of statistic of nongaussian processes. In present work we consider a class of nongaussian distributions in the space of nonlinear functionals associated with processes determined by signed measures defined on function spaces. These measures are of unbounded variation but their characteristic functional have simple structure, that allows to carry out exact evaluations. We give some characterization the reproducing Hilbert space of measure $\widehat{\mu}$, and in two special cases we get orthonormal basis of this space. Note that since we deal with functionals of stochastic process $\xi(t)=\xi(t, \omega), t \in T, \omega \in(\Omega, P)$ it is convenient for us to use instead of $P$ the corresponding distributions in functional space $X$ of functions $x(t), t \in T$, with $\sigma$-algebra $\mathcal{B}$ generated by cylindrical sets. In this case we can use the formula

$$
\mathbb{E} f(\xi(\cdot))=\int_{X} f(x(\cdot)) d \mu_{\xi}(x(\cdot)),
$$

where $\mathbb{E}$ be the symbol of mathematical expectation, $\mu_{\xi}$ - the distribution defined by formula $\mu_{\xi}(A)=P\{\xi(\cdot) \in A\}, A \in \mathcal{B}$.

So, let $\gamma$ is a Gaussian measure on linear space $X$ with zero mean value and covariance functional $K(\xi, \eta), \xi, \eta \in X^{\prime}$. The next two Hilbert spaces (see, for example, Egorov et al., 1993) are used widely in the theory of Gaussian distributions. The space $H \equiv H_{\gamma}$ is the closure of the set of linear functional $\langle\xi, \cdot\rangle \quad\left(\xi \in X^{\prime}\right)$ in the space $L_{2}(X, \gamma)$, and the Hilbert space $\mathcal{H} \subset X$ is dual to $H$. The space $H$ is known as the reproducing Hilbert 
space of the measure $\mu$, and $\mathcal{H}$ is called the the Cameron-Martin space. For almost all $x \in X$ the next series is defined: $(a, x)=\sum_{k=1}^{\infty}\left\langle\varphi_{k}, x\right\rangle\left(a, e_{k}\right)_{\mathcal{H}}$, where $\left\{\varphi_{k}\right\},\left\{e_{k}\right\}$, $k=1,2, \ldots$, are orthonormal bases in $H$ and $\mathcal{H}$, respectively, with $\varphi_{k} \in X^{\prime}$ for all $k$ and $\left\langle\varphi_{k}, e_{j}\right\rangle=\delta_{k j}$; the functional $(a, x)$ is called measurable linear functional. There exists an isomorphism $T$ of $\mathcal{H}$ into $H$ which assigns a basis $\left\{\varphi_{k}\right\}$ to the basis $\left\{e_{k}\right\}$. So one have $\langle\xi, x\rangle=\left(T^{-1} \xi, x\right)$ and $K(\xi, \eta)=(\xi, \eta)_{H}=\left(T^{-1} \xi, T^{-1} \eta\right)_{\mathcal{H}}$. It is known that arbitrary functional $f(x) \in L_{2}(X, \gamma)$ can be presented in the form of functional Fourier Hermite series

$$
\begin{gathered}
\varphi(x)=\sum_{n=0}^{\infty} \sum_{\alpha \in I^{n}} a_{\alpha} H_{\alpha}(x), \\
a_{\alpha}=\frac{1}{n(\alpha) !} \int_{X} \varphi(x) H_{\alpha}(x) d \gamma(x),
\end{gathered}
$$

where $H_{\alpha}(x) \equiv: \prod_{k=1}^{n}\left\langle\varphi_{\alpha_{k}}, x\right\rangle:_{\gamma} \quad$ denotes the functional Hermite polynomial (Wick's monomial). Wick's monomials form orthogonal basis in $L_{2}(X, \gamma)$ and can be received by differentiation the Wick's exponent

$$
: e^{\langle\xi, x\rangle}:_{\gamma}=e^{\langle\xi, x\rangle-1 / 2 K(\xi, \xi)}=\sum_{n=0}^{\infty} \frac{1}{n !}:\langle\xi, x\rangle^{n}:_{\gamma} \equiv \widehat{\xi}(x) .
$$

\section{Gaussian Constructions Connected with Signed Mea- sure}

A nongaussian signed measure $\mu$ which we consider here defined by the characteristic functional

$$
\chi_{\mu}(\xi)=\int_{X} \exp \{i\langle\xi, x\rangle\} d \mu(x)=\exp \left\{\frac{(-1)^{p}}{(2 p) !} K_{2 p}(\xi, \stackrel{(2 p)}{.}, \xi)\right\}
$$

where $K_{2 p}(\xi, \stackrel{(2 p)}{.}, \xi)$ is a symmetric $2 p$-linear form on $X^{\prime} \times \stackrel{(2 p)}{.} \times X^{\prime}$. The above mentioned Gaussian measure $\widehat{\mu}$ on $Y$ with zero average value is defined by its correlation functional

$$
\widehat{K}(f, g)=\int_{X} \int_{X} \widehat{\mathcal{K}}\left(x_{1}, x_{2}\right) f\left(x_{1}\right) g\left(x_{2}\right) d \gamma\left(x_{1}\right) d \gamma\left(x_{2}\right),
$$

where

$$
\begin{gathered}
\widehat{\mathcal{K}}\left(x_{1}, x_{2}\right)=\sum_{r=1}^{2 p-1}(r !(2 p-r) !)^{-1} \sum_{\substack{i_{1}, \ldots, i_{r}=1 \\
j_{1}, \ldots, j_{2 p}-r=1}}^{\infty} K_{2 p}\left(\varphi_{i_{1}}, . r\right), \varphi_{i_{r}}, \\
\left.\varphi_{j_{1}}, \stackrel{(2 p-r)}{ }, \varphi_{j_{2 p-r}}\right): \prod_{l_{1}=1}^{r}\left\langle\varphi_{i_{l_{1}}}, x_{1}\right\rangle:_{\gamma}: \prod_{l_{2}=1}^{2 p-r}\left\langle\varphi_{j_{l_{2}}}, x_{2}\right\rangle:_{\gamma} .
\end{gathered}
$$


Using the properties of Hermite polynomials we get

$$
\widehat{K}(\Xi, \Psi)=K_{2 p}\left(\xi_{1}, \ldots, \xi_{n}, \psi_{1}, \ldots, \psi_{m}\right), \quad n+m=2 p,
$$

where $\Xi=: \prod_{l=1}^{n}\left\langle\xi_{l}, x\right\rangle:_{\gamma}, \Psi=: \prod_{l=1}^{m}\left\langle\psi_{l}, x\right\rangle:_{\gamma}, \xi_{l}, \psi_{l} \in X^{\prime} ;$ and $\widehat{K}(\Xi, \Psi)=0$ for $n+m \neq 2 p$.

Let us define $\mu$ - and $\widehat{\mu}$-exponentials

$$
: e^{\langle\xi, x\rangle}:_{\mu}=\exp \left\{\langle\xi, x\rangle-\frac{(-1)^{p}}{(2 p) !} K_{2 p}(\xi, \stackrel{(2 p)}{.}, \xi)\right\}, \quad: e^{\langle\widehat{\xi}, y\rangle}: \widehat{\mu}=\exp \left\{\langle\widehat{\xi}, y\rangle-\frac{1}{2} \widehat{K}(\widehat{\xi}, \widehat{\xi})\right\}
$$

and consider the map

$$
\widehat{S}: e^{\langle\xi, x\rangle}: \mu \rightarrow: e^{\langle\widehat{\xi}, y\rangle}: \widehat{\mu}, \quad x \in X, y \in Y
$$

The following equality take place

$$
\left\langle\widehat{S}\left(: e^{\langle\xi, \cdot\rangle}:_{\mu}\right), \widehat{S}\left(: e^{\langle\eta, \cdot\rangle}:_{\mu}\right)\right\rangle_{L_{2}(Y, \widehat{\mu})}=\left\langle: e^{\langle\xi, \cdot}:_{\mu},: e^{\langle\eta, \cdot\rangle}:_{\mu}\right\rangle_{L_{2}(X, \mu)} .
$$

It can be proved by using explicit form of characteristic functionals of measures $\mu$ and $\widehat{\mu}$. As $\mu$ - and $\widehat{\mu}$-exponentials are generating functionals of Appel polynomials (Wick's polynomials in case of Gaussian measures), by differentiation it is possible to receive $\widehat{S}$ images of the relevant polynomials and extend $\widehat{S}$ on their linear envelop. The formula (2) can used to obtain the next equality valid at least for functional polynomials of $x \in X$

$$
\begin{aligned}
\int_{X} f(x) & : e^{\langle\xi, x\rangle}:_{\mu} d \mu(x)=\int_{Y}(\widehat{S} f)\left(y-a_{\widehat{\xi}}\right) d \widehat{\mu}(y)= \\
& =\int_{X} \widehat{S}^{-1}\left[(\widehat{S} f)\left(\cdot-a_{\widehat{\xi}}\right)\right](x) d \mu(x),
\end{aligned}
$$

where $a_{\widehat{\xi}}=\widehat{T}^{-1} \widehat{\xi}$ and the map $\widehat{T}^{-1}$ is defined by the kernel $\widehat{\mathcal{K}}\left(x_{1}, x_{2}\right)$.

By virtue of polarization identity for symmetric multilinear forms, it is sufficient to define correlation functional on functions of the form : $\langle\xi, x\rangle^{n}:_{\gamma}, n=1,2, \ldots$ Therefore, in what follows we will only consider the linear functionals on $Y$ of the form

$$
\Xi \equiv \Xi(x)=\sum_{n=0}^{\infty}:\left\langle\xi_{n}, x\right\rangle^{n}:_{\gamma}, \quad \xi_{n} \in X^{\prime}, n=1,2, \ldots
$$

Note that $\widehat{K}(\Xi, \Psi)=0$ if the functionals $\Xi, \Psi$ include only Wick's monomials of degree $\geq 2 p$, so the summation in (3) is taken over $n=1,2, \ldots, 2 p-1$.

Let us define the space $\widehat{H}=H_{\widehat{\mu}}$. For this goal we define symmetric bilinear form in $Y^{\prime}$ by equality $(\Xi, \Psi)_{\widehat{H}}=\widehat{K}(\Xi, \Psi)$. Then we denote $\widehat{H}_{r}$ the linear envelope of functionals

$$
\Xi(x)=:\left\langle\xi_{r}, x\right\rangle:_{\gamma}^{r}+:\left\langle\xi_{2 p-r}, x\right\rangle:_{\gamma}^{2 p-r}, \quad r=\overline{1, p},
$$


where both $\xi_{r}$ and $\xi_{2 p-r}$ are not equal to zero. The functionals (4) for different $r$ are orthogonal in $L_{2}(Y, \widehat{\mu})$-metrics and

$$
\begin{gathered}
(\Xi, \Psi)_{\widehat{H}}=K\left(\xi_{r}, \stackrel{(r)}{.}, \xi_{r}, \psi_{2 p-r}, \stackrel{(2 p-r)}{\cdot .}, \psi_{2 p-r}\right)+K\left(\psi_{r}, \stackrel{(r)}{\cdot}, \psi_{r}, \xi_{2 p-r}, \stackrel{(2 p-r)}{\cdot}, \xi_{2 p-r}\right), \\
\|\Xi\|_{\widehat{H}}^{2} \equiv \int_{Y}(\Xi, y)^{2} d \widehat{\mu}(u)=2 K\left(\xi_{r}, \stackrel{(r)}{\cdot}, \xi_{r}, \xi_{2 p-r}, \stackrel{(2 p-r)}{\cdots}, \xi_{2 p-r}\right) .
\end{gathered}
$$

The bilinear form $(\Xi, \Psi)_{\widehat{H}}$ doesn't define a norm in $Y$, because there exist elements $\Xi \in$ $Y^{\prime}$ such that $\|\Xi\|_{\widehat{H}}^{2}<0$. We suppose also that the measure $\widehat{\mu}$ is nondegenerate, that is $\|\Xi\|_{\widehat{H}}^{2}=0$ only if $\Xi=0$. By virtue of nondegeneracy of the norm (6) the space $\widehat{H}_{r}$ can be presented as orthogonal sum of subspaces $\widehat{H}_{r+}$ and $\widehat{H}_{r-}$ of elements with positive and negative norm respectively:

$$
\widehat{H}_{r}=\widehat{H}_{r+}+\widehat{H}_{r-} .
$$

We will use the same notations for completion of these spaces. Because of orthogonality of functional (4) under different $r$, we can define the space:

$$
\widehat{H}=\sum_{r=1}^{p} \widehat{H}_{r}=\sum_{r=1}^{p}\left(\widehat{H}_{r+}+\widehat{H}_{r-}\right) .
$$

If we obtain orthogonal basis in $\widehat{H}$ we can write orthogonal system of Hermite polynomial in $L_{2}(Y, \widehat{\mu})$-metrics. Note that in fact the space $\widehat{H}$ is defined here as linear manifold, and the question of completion of this space in some topology require special development.

\section{Orthonormal Basis in $\widehat{H}$}

Consider two examples when the basis in the space $\widehat{H}_{r+}$ ( respectively $\widehat{H}_{r-}$ ) may be received in explicit form.

1. Let $X=\mathbb{C}[0,1]$ be the space of continuous functions and

$$
\widehat{K}_{2 p}\left(\xi_{1}, \ldots, \xi_{2 p}\right)=\int_{0}^{1}(2 p) \int_{0}^{1} \min \left(t_{1}, \ldots, t_{2 p}\right) d \xi_{1}\left(t_{1}\right) \ldots d \xi_{2 p}\left(t_{2 p}\right)
$$

where $\xi_{i}(t), i=1, \ldots, 2 p$, are the functions of bounded variation (see Hochberg, 1978, for corresponding signed measure $\mu$ ). into

Since $\widehat{K}_{2 p}\left(\xi_{1}, \ldots, \xi_{2 p}\right)=\int_{0}^{1} \xi_{1}(u) \ldots \xi_{2 p}(u) d u$, the equality (5) can be transformed

$$
(\Xi, \Psi)_{\widehat{H}}=\int_{0}^{1}\left[\xi_{r}^{r}(u) \psi_{2 p-r}^{2 p-r}(u)+\psi_{r}^{r}(u) \xi_{2 p-r}^{2 p-r}(u)\right] d u
$$

Let $\left\{\alpha_{k} \equiv \alpha_{k}(t), k=1,2, \ldots\right\}$ be orthonormal basis in $L_{2}[0,1] ; \quad \alpha_{k}^{\frac{1}{r}}=\left(\alpha_{k}(t)\right)^{\frac{1}{r}}$. Then the set

$$
A_{k}^{(r)}(x)=\frac{1}{\sqrt{2}}\left[:\left\langle\alpha_{k}^{\frac{1}{r}}, x\right\rangle^{r}:_{\gamma}+:\left\langle\alpha_{k}^{\frac{1}{2 p-r}}, x\right\rangle^{2 p-r}:_{\gamma}\right], \quad k=1,2, \ldots
$$


is the orthonormal basis in $\widehat{H}_{r+}$. In fact, substituting (7) into (5), we obtain

$$
\begin{gathered}
\left(A_{k}^{(r)}, A_{j}^{(r)}\right)_{\widehat{H}}=\int_{0}^{1} \alpha_{k}(u) \alpha_{j}(u) d u=\delta_{k j}, \\
\sum_{k=1}^{\infty}\left(\Xi, A_{k}^{(r)}\right)^{2}=\sum_{k=1}^{\infty}\left(\int_{0}^{1}\left[\xi_{r}^{r}(u)+\xi_{2 p-r}^{2 p-r}(u)\right] \alpha_{k}(u) d u\right)^{2}=\left\|\xi_{r}^{r}+\xi_{2 p-r}^{2 p-r}\right\|_{L_{2}[0,1]}^{2} .
\end{gathered}
$$

Taking into account $\xi_{r}(u) \neq 0, \xi_{2 p-r} \neq 0, \int_{0}^{1} \xi_{r}^{2 p}(u) d u<\infty, \int_{0}^{1} \xi_{2 p-r}^{(2 p-r)^{2}}(u) d u<\infty, r=$ $\overline{1, p}$ and completeness of $\left\{\alpha_{k}(u)\right\}, k=1,2, \ldots$, in $L_{2}[0,1]$, one gets from these equalities the statement.

2. Now let

$$
\widehat{K}_{2 p}\left(\xi_{1}, \ldots, \xi_{2 p}\right)=\sum_{k=1}^{\infty} \lambda_{k} \prod_{l=1}^{2 p}\left(\xi_{l}, \varphi_{k}\right)_{H_{\gamma}}
$$

where $\left\{\varphi_{k}\right\}_{k=1,2, \ldots}$ is orthonormal basis in $H_{\gamma}$ and

$$
\sum \lambda_{k}\left(\xi_{l}, \varphi_{k}\right)^{4 p-2}<\infty, l=\overline{1,2 p}
$$

Then we have

$$
(\Xi, \Psi)_{\widehat{H}}=\sum_{j=1}^{\infty} \lambda_{j}\left[\left(\xi_{r}, \varphi_{j}\right)_{H_{\gamma}}^{r}\left(\psi_{2 p-r}, \varphi_{j}\right)_{H_{\gamma}}^{2 p-r}+\left(\psi_{r}, \varphi_{j}\right)_{H_{\gamma}}^{r}\left(\xi_{2 p-r}, \varphi_{j}\right)_{H_{\gamma}}^{2 p-r}\right], \quad r=\overline{1, p}
$$

It is not difficult to verify that the set

$$
A_{k}^{(r)}=\frac{1}{\sqrt{2}}\left[:\left\langle\lambda_{k}^{-\frac{1}{2 r}} \varphi_{k}, x\right\rangle^{r}:_{\gamma}+:\left\langle\lambda_{k}^{-\frac{1}{2(2 p-r)}} \varphi_{k}, x\right\rangle^{2 p-r}:_{\gamma}\right], \quad k=1,2, \ldots,
$$

is the orthonormal basis in $\widehat{H}_{r+}$. Really, substituting (10) into (9) we get:

$$
\begin{gathered}
\left(A_{k_{1}}^{(r)}, A_{k_{2}}^{(r)}\right)_{\widehat{H}}=\sum_{j=1}^{\infty} \frac{1}{2} \lambda_{j}\left[\left(\lambda_{k_{1}}^{-\frac{1}{2 r}} \varphi_{k_{1}}, \varphi_{j}\right)_{H_{\gamma}}^{r}\left(\lambda_{k_{2}}^{-\frac{1}{2(2 p-r)}} \varphi_{k_{2}}, \varphi_{j}\right)_{H_{\gamma}}^{2 p-r}+\right. \\
\left.\quad+\left(\lambda_{k_{1}}^{-\frac{1}{2 r}} \varphi_{k_{2}}, \varphi_{j}\right)_{H_{\gamma}}\left(\lambda_{k_{2}}^{-\frac{1}{2(2 p-r)}} \varphi_{k_{1}}, \varphi_{j}\right)_{H_{\gamma}}\right]=\delta_{k_{1} k_{2}} \\
\sum_{k=1}^{\infty}\left(\Xi, A_{k}^{(r)}\right)_{\widehat{K}}^{2}=\sum_{k=1}^{\infty}\left[\left(\xi_{r}, \varphi_{k}\right)_{H_{\gamma}}^{r}+\left(\xi_{2 p-r}, \varphi_{k}\right)_{H_{\gamma}}^{2 p-r}\right]^{2}<\infty
\end{gathered}
$$

The last inequality is valid due to (8).

Note that basis in $\widehat{H}_{r-}$ can be obtained by change of sign in front of the second term in (7) and (10). 


\section{References}

A.D. Egorov. On $\mathrm{L}_{2}$-isomorphic gaussian models for nongaussian distributions. Monte Carlo Methods and Appl., 3(2):485-490, 1997.

A.D. Egorov, P.I. Sobolevsky, and L.A. Yanovich. Functional integrals: Approximate evaluation and Applications. Kluwer Academic Publishers, Dordrecht, 1993.

K.J. Hochberg. A signed measure on path space related to wiener measure. The Annals of Probability, 6(3):433-458, 1978.

Yu.A. Rozanov. Gaussian infinite-dimensional distributions (in russian). Trudy Matem. Inst. V.A.Steklova, 108, 1968.

Authors' addresses:

Prof. Dr. A.D. Egorov

Department of Nonlinear and Stochastic Analysis

National Academy of Sciences

Institute of Mathematics

Surganova str., 11

Minsk

Belarus

Tel. +375 17 284-15-55

Fax. +375 17 284-09-15

E-mail: egorov@im.bas-net.by

A.V. Zherelo

Department of Nonlinear and Stochastic Analysis

National Academy of Sciences

Institute of Mathematics

Surganova str., 11

Minsk

Belarus

Tel. +375 17 284-19-69

Fax. +375 17 284-09-15

E-mail: ant@im.bas-net.by 